OPEN ACCESS

Edited by:

Steven W. Howard,

Saint Louis University, United States

Reviewed by:

Rebecca Thorsness,

Brown University, United States

Alyssa Coleman

Saint Louis University, United States

${ }^{*}$ Correspondence:

Cyrus Ayubcha

cyrusayubcha@hms.harvard.edu

Specialty section:

This article was submitted to

Public Health Policy,

a section of the journal

Frontiers in Public Health

Received: 05 October 2021

Accepted: 08 October 2021

Published: 17 November 2021

Citation:

Ayubcha $C$, Pouladvand $P$ and Ayubcha S (2021) A

Quasi-Experimental Study of Medicaid Expansion and Urban Mortality in the

American Northeast.

Front. Public Health 9:707907.

doi: 10.3389/fpubh.2021.707907

\section{A Quasi-Experimental Study of Medicaid Expansion and Urban Mortality in the American Northeast}

\author{
Cyrus Ayubcha ${ }^{1 *}$, Pedram Pouladvand ${ }^{2}$ and Soussan Ayubcha ${ }^{3}$ \\ ${ }^{1}$ Harvard Medical School, Boston, MA, United States, ${ }^{2}$ Alfred I. DuPont Hospital for Children, Wilmington, NC, \\ United States, ${ }^{3}$ Marcus Institute of Integrative Health, Thomas Jefferson University, Philadelphia, PA, United States
}

Objectives: To investigate the association of state-level Medicaid expansion and nonelderly mortality rates from 1999 to 2018 in Northeastern urban settings.

Methods: This quasi-experimental study utilized a synthetic control method to assess the association of Medicaid expansion on non-elderly urban mortality rates [1999-2018]. Counties encompassing the largest cities in the Northeastern Megalopolis (Washington D.C., Baltimore, Philadelphia, New York City, and Boston) were selected as treatment units ( $n=5$ cities, 3,543,302 individuals in 2018). Cities in states without Medicaid expansion were utilized as control units ( $n=17$ cities, 12,713,768 individuals in 2018).

Results: Across all cities, there was a significant reduction in the neoplasm (Population-Adjusted Average Treatment Effect $=-1.37$ [95\% Cl $-2.73,-0.42]$ ) and all-cause (Population-Adjusted Average Treatment Effect $=-2.57[95 \% \mathrm{Cl}-8.46$, -0.58]) mortality rate. Washington D.C. encountered the largest reductions in mortality (Average Treatment Effect on All-Cause Medical Mortality $=-5.40$ monthly deaths per 100,000 individuals [95\% Cl $-12.50,-3.34],-18.84 \%$ [95\% Cl $-43.64 \%,-11.67 \%]$ reduction, $p=<0.001$; Average Treatment Effect on Neoplasm Mortality $=-1.95$ monthly deaths per 100,000 individuals $[95 \% \mathrm{Cl}-3.04,-0.98],-21.88 \%[95 \% \mathrm{Cl}$ $-34.10 \%,-10.99 \%]$ reduction, $p=0.002$ ). Reductions in all-cause medical mortality and neoplasm mortality rates were similarly observed in other cities.

Conclusion: Significant reductions in urban mortality rates were associated with Medicaid expansion. Our study suggests that Medicaid expansion saved lives in the observed urban settings.

Keywords: Medicaid expansion, Medicaid, cities, mortality, urban

\section{INTRODUCTION}

The Affordable Care Act (ACA) offered states the opportunity to expand health insurance coverage to non-elderly adult populations through Medicaid expansion (ME). States were able to use federal funding to increase state Medicaid coverage to all those US Citizens and permanent residents with incomes at or below $138 \%$ federal poverty level (FPL) (1). Specific narrow categories of eligibility (e.g., impoverished pregnant women) were federally mandated earlier. Prior to the 2014 implementation of ACA Medicaid expansion, some states utilized waivers to preemptively expand their programs sometimes with more expansive eligibility criteria, but significant gaps in coverage 
persisted (2). As a result of Medicaid expansion, Medicaid take-up increased in less-educated, low-income, minority, and younger adults residing in expansion states as compared to peers in nonexpanded states (3). What remains unclear is whether this increase in coverage improved health outcomes, particularly whether urban settings observed reductions in mortality.

Cost-benefit considerations, entailing monetary cost, value of increasing coverage, and the quality of care provided to beneficiaries, have been of notable interest in the debate to increase public medical coverage (4). The original Medicaid program has been associated with moderate decreases in mortality depending on the methods employed (5). Expansions of Medicaid to pregnant women and children during the $1980 \mathrm{~s}$ were linked to decreases in infant mortality and maternal mortality in most studies (6, 7). Analyses of the 2006 Massachusetts Health Care Reform (MHCR) found reduced all-cause mortality by nearly 8.2 deaths per 100,000 adults (8-10). However, the Oregon Healthcare Experiment study suggested that the effects of coverage may not be immediate or large (11). Such variability may suggest the mechanisms and impact of health coverage is contingent upon alternative factors (e.g., urban-rural residence, minority identity, socioeconomic status, etc.); specific benefits to certain sub-populations or temporally removed effects may further underlie such variability. Smaller mortality effects may also be challenging to capture as gains in public insurance can often be centered in younger populations though older populations are most likely to benefit from coverage.

A number of studies have examined the ACA Medicaid expansion directly and these studies also gesture toward effect heterogeneity in increasing coverage. Nationwide studies observed a decline in all-cause mortality following the ACA Medicaid expansions, but not in cause-specific mortality rates (cardiovascular, respiratory, suicide, and opioid overdose) (12) while a separate analysis found only small, insignificant effects (13). Emerging evidence suggests that Medicaid expansion may have reduced excessive mortality for minorities, in part, by reducing amenable mortality $(10,14)$. These reforms also seem to have reduced maternal mortality rates, particularly for latematernal deaths and Black mothers $(15,16)$. Some studies have observed improvements in specific mortality measures, such as cardiovascular mortality in near-elderly populations and oneyear mortality rates among end-stage renal disease patients (17, 18).

We examine the influence of Medicaid expansion on the mortality rates in various Northeastern urban centers. Urban populations warrant targeted study as they are largely distinct from state-wide populations with respect to diversity, healthcare access barriers, types of disease burden and disparities; this is not to mention that a majority of Americans reside in urban settings (19-24). Notably, most large American metropolitan centers are unique in characteristics and state policy history which presents difficulties in studying the generalized "urban" populations. This motivates our study to take a narrow and detailed examination into each city to determine the impact of Medicaid expansion. Accordingly, the observed changes across the included cities may be a means to logically deducing certain city-specific environmental factors that underlie treatment effects. Our study does not only examine all-cause medical mortality but also certain cause-specific mortality rates (e.g., circulatory mortality, neoplasm mortality, etc.). This study will help to understand whether expanding medical coverage can reduce all-cause and cause-specific urban mortality rates, thus helping illuminate why health coverage may improve health for some but not all.

\section{MATERIALS AND METHODS Study Design and Inclusion Criteria}

We utilized a quasi-experimental design to assess the mortality rate among those aged 20-64 from 1999 to 2018; where observed mortality rates after Medicaid expansion were compared to respective predicted mortality rates for each treated city. Only cities in the Northeast megalopolis were considered when selecting treated urban counties. The first year of treatment for each treated city unit was considered the year in which statewide Medicaid expansion was enacted. Urban counties within non-expansion states were selected for the control group. All urban counties with sufficient population levels $(>9,000$ individuals) and population density and (>800 individuals/mi2) were included. States and counties with any previous Federal Poverty Level (FPL)-based waiver expansions were eliminated from this control pool (e.g., Wisconsin, St. Louis City, MO). The counties utilized in this study can be found in Table $\mathbf{1}$.

\section{Data Sources}

All data were secondary, public, and de-identified; no institutional review board approval or informed consent was required. County-level age-adjusted mortality data for individuals between the ages of 20-64 were compiled from the Centers for Disease Control WONDER Tool. The following categories of mortality were included: diseases of the circulatory system mortality (circulatory mortality), diseases of the respiratory system mortality (respiratory mortality), all-cause medical mortality, and neoplasm mortality (i.e., cancer, malignancy). All-cause medical mortality was defined as allcause mortality absent external-cause mortality. Categories of mortality were defined by International Classification of Diseases (ICD) coding systems; the ICD 9 to ICD 10 code transition was reconciled.

Several longitudinal county-level covariates were obtained and utilized as the basis for developing the synthetic control for each treatment city. From 1999 to 2018, healthcare coverage rates were attained from the Small Area Health Insurance Estimates, non-Hispanic white percentages of populations between 20 and 64 were calculated using the Bridged-Race Population Estimates, inflation-adjusted median income and poverty rates were captured in data from the Bureau of Economic Analysis and the Small Area Income and Poverty Estimates. The Economic Research Services of the United States Department of Agriculture provided educational attainment fractions as defined as the fraction of those with at least some college. 
TABLE 1 | Treatment conditions of included cities.

\begin{tabular}{|c|c|c|c|c|c|}
\hline City & County & State & Expansion year & Expansion FPL & Treatment \\
\hline Baltimore $^{\dagger}$ & Baltimore City & MD & 2014 & $138 \%$ & Treatment \\
\hline San Antonio & Bexar & TX & None & None & Control \\
\hline Fort Lauderdale & Broward & $\mathrm{FL}$ & None & None & Control \\
\hline Dallas & Dallas & TX & None & None & Control \\
\hline Nashville & Davidson & $\mathrm{TN}$ & None & None & Control \\
\hline Washington D.C. ${ }^{\dagger \dagger}$ & District of Columbia & & 2010 & $210 \%$ & Treatment \\
\hline Atlanta & Fulton & GA & None & None & Control \\
\hline Houston & Harris & TX & None & None & Control \\
\hline Kansas City & Johnson & KS & None & None & Control \\
\hline Charlotte & Mecklenburg & $\mathrm{NC}$ & None & None & Control \\
\hline Memphis & Shelby & $\mathrm{TN}$ & None & None & Control \\
\hline Miami & Miami-Dade & $\mathrm{FL}$ & None & None & Control \\
\hline New York City ${ }^{\ddagger}$ & New York & NY & 2014 & $138 \%$ & Treatment \\
\hline Oklahoma City & Oklahoma & OK & None & None & Control \\
\hline Orlando & Orange & $\mathrm{FL}$ & None & None & Control \\
\hline Philadelphia & Philadelphia & PA & 2015 & $138 \%$ & Treatment \\
\hline Tampa St. Petersburg Clearwater & Pinellas & $\mathrm{FL}$ & None & None & Control \\
\hline Salt Lake City & Salt Lake & UT & None & None & Control \\
\hline Boston ${ }^{\ddagger \ddagger}$ & Suffolk & MA & 2014 & $138 \%$ & Treatment \\
\hline Fort Worth & Tarrant & TX & None & None & Control \\
\hline Austin & Travis & TX & None & None & Control \\
\hline Raleigh & Wake & $\mathrm{NC}$ & None & None & Control \\
\hline
\end{tabular}

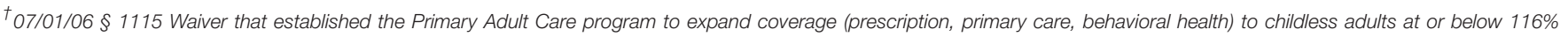
of FPL.

†† 07/01/10 State Plan Amendment extends Medicaid coverage to 133\% FPL | 12/01/10 \$ 1115 Waiver Early ACA expansion extends Medicaid program to 210\% of FPL.

$\$ 10 / 01 / 01$ \$ 1115 Waiver extends Medicaid Family Health Plus to childless adults at $100 \%$ FPL.

$\neq \neq$ 4/6/06 Massachusetts implemented reforms to expand insurance coverage to low-income adults beginning in 2006.

Unavailable covariate data for health insurance coverage (19992005) were interpolated using only the trends provided by fiveyear American Community Survey estimates and Census data.

\section{Main Data Outcomes}

Mortality was acquired from 1999 to 2018 and all rates were calculated as age-adjusted deaths per 100,000 population of 2064 -year-olds in the county. Monthly data were used for all-cause medical mortality and neoplasm mortality rates, while yearly data were used for all other forms of mortality; more granular data (i.e., monthly as opposed to yearly) provided greater statistical power but less common forms of mortality lacked sufficient prevalence to justify monthly analyses in light of limited sample sizes and elevated risk of stochasticity.

\section{Statistical Analysis}

This study employs synthetic control methods which are advantageous when no single control unit can serve as an ideal comparator for a treated unit. This application creates a synthetic control city unit through a weighted combination of the control cities. The synthetic city is intended to simulate the mortality rate of the treated city (e.g., Philadelphia) in the post-treatment period (e.g., 2015-2018) if Medicaid had not been expanded. The particular weights varied in the specific model as applied to each treated city; selection of weights chiefly aims to create a synthetic city with similar pre-intervention covariate characteristics (e.g., inflation-adjusted median income, lagged mortality rates) to the treated city; this algorithmic process employed by the GSC is akin to creating a control city with similar characteristics to the treated city. The appropriateness of the synthetic city as a comparator to the treated city is determined by the convergence of the pre-intervention outcome trends between the treated city and the synthetic city. Such that, any divergence of trends in the post-intervention can be attributed to the intervention (i.e., Medicaid expansion).

In this study, we utilize a Generalized Synthetic Control (GSC) model (25) for each treated city and specific mortality rate. The GSC model relies upon an interactive fixed effects (IFE) technique within the synthetic control framework. The use of IFE incorporates two-way fixed effects which enabled our model to account for unobserved unit-specific and time-specific confounding variables. IFE modeling of the control units is then used to create out-of-sample predictions for the treated unit which results in a GSC output of the synthetic city's mortality rates. The difference between the synthetic and treatment mortality outcomes is considered the treatment effect on the treated unit. The post-intervention difference between the trends can be 
averaged over the post-treatment period; termed the average treatment effected in the treated units (ATT). Parametric bootstrapping inference tests were applied to ascertain uncertainty estimates (25).

One sensitivity test was performed by rerunning the models of the monthly data in yearly format to determine whether the structure of yearly analyses influenced the model. An intime placebo sensitivity analysis was performed applying a false intervention point in the middle of the pre-intervention period and running the GSC model through the pre-intervention period; specifically, the pre-treatment period was considered 1999-2005 and the post-treatment period was considered 2006-2007 so that the 2008 economic downturn was not included. Given that insurance rate is considered a significant mediating variable, a secondary analysis was also performed utilizing county-level insurance rates for each of the treatment cities. The GSC model was applied to insurance coverage rates as a secondary outcome using all the predictors utilized in the main models except for mortality data. All statistical analyses were conducted in $\mathrm{R}$ (version 4.0.0) using the gsynth package.

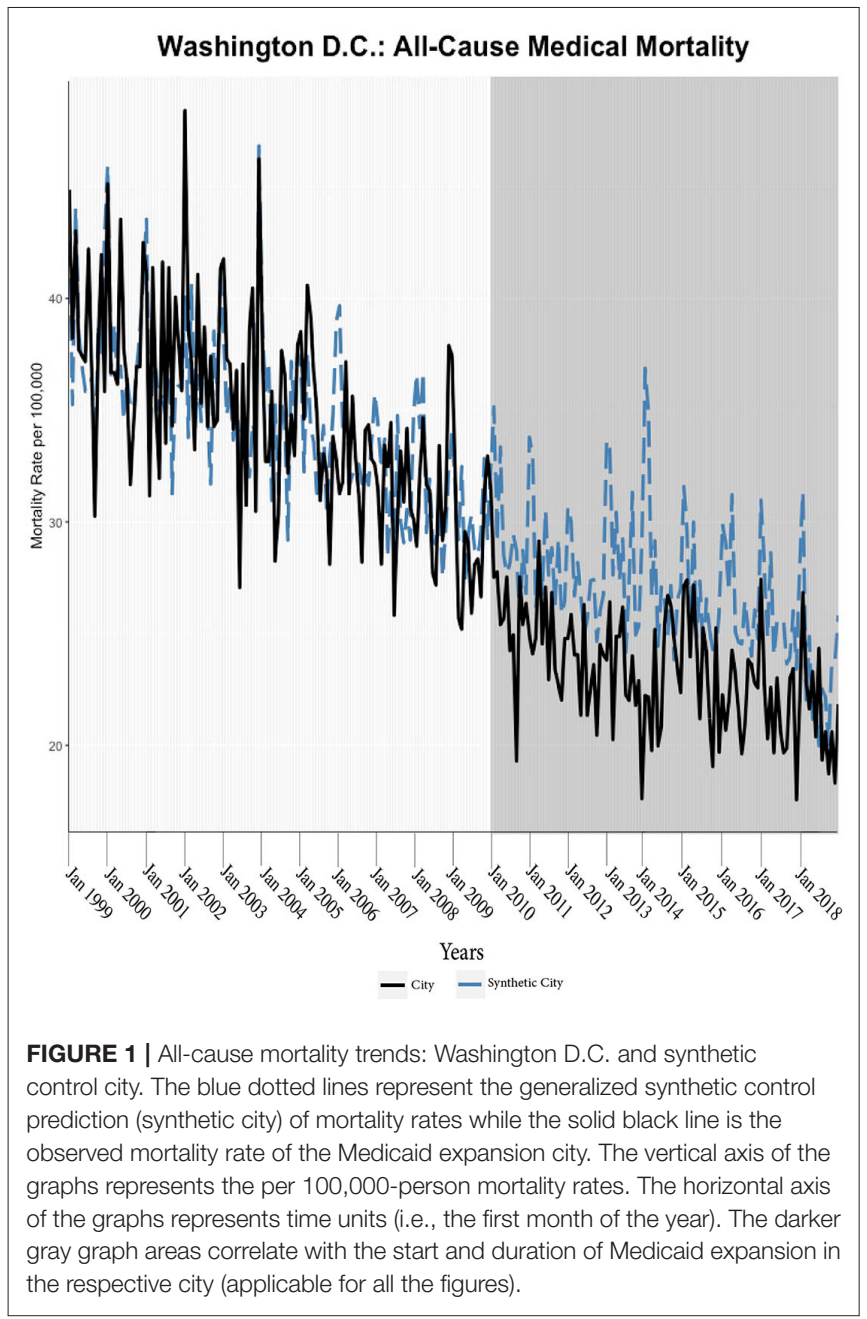

The GSC overcomes some limitations of alternative methods. The GSC does not rely upon the parallel trend assumption that is required in the difference-in-differences methods (25). The GSC method has further proven to be less sensitive to idiosyncratic volatility with a small number of observations (25). Furthermore, this method does not require sensitivity tests of model specifications as a cross-validation procedure selects the optimal number of factors in the IFE model (25). Nevertheless, the GSC model does assume that a stable combination of control units based on the pre-intervention period characteristics of the treatment and control pool can approximate the outcomes of the treated unit in the post-intervention period absent the intervention. Such advantages may achieve similar or superior performance as compared to other methods; several simulated and applied health policy studies comparing IFE, GSC, DiD, and synthetic control methods have found that GSC models perform best $(25,26)$. Finally, the GSC structure allows for robust parametric bootstrapping inference whereas such quantitative inference is unavailable for traditional synthetic control methods.

\section{RESULTS}

We found evidence of significant reductions in all-cause medical mortality as compared to predicted mortality had expansion not occurred in four cities, including Washington D.C., Baltimore, Philadelphia, and New York, after their 2010, 2014, 2015, and 2014 expansions of Medicaid, respectively (Figures 1-5). Similar

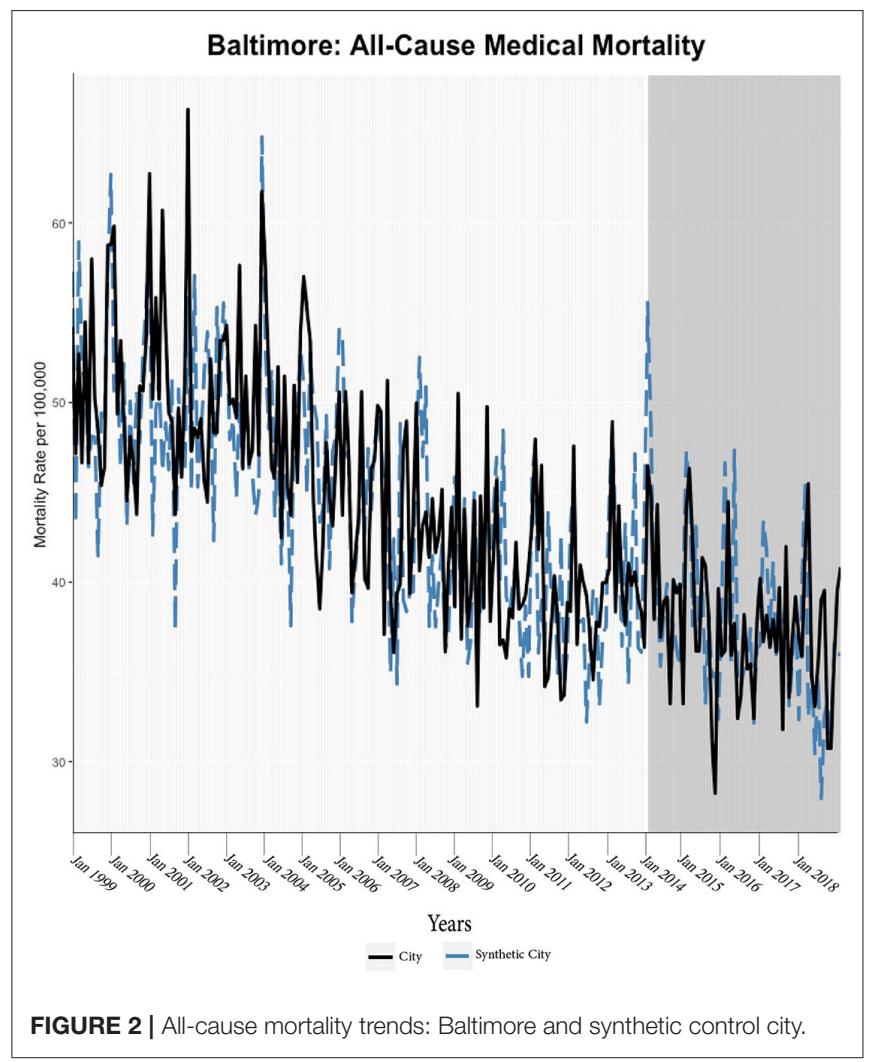




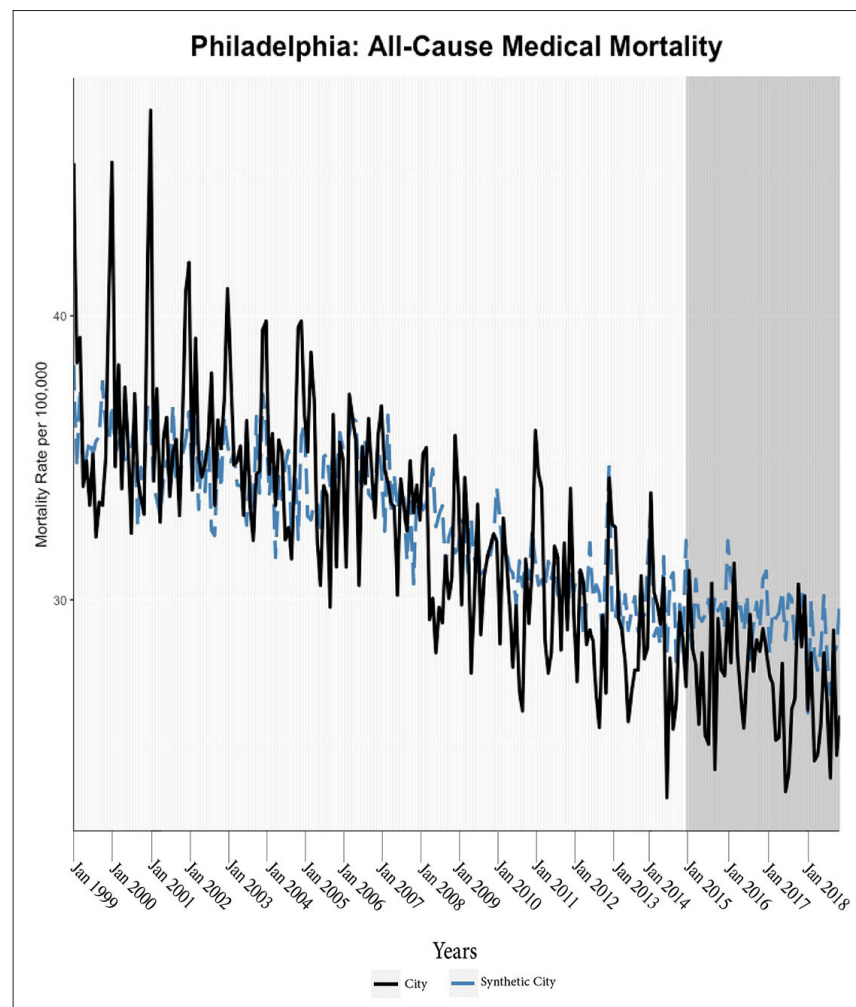

FIGURE 3 | All-cause mortality trends: Philadelphia and synthetic control city.

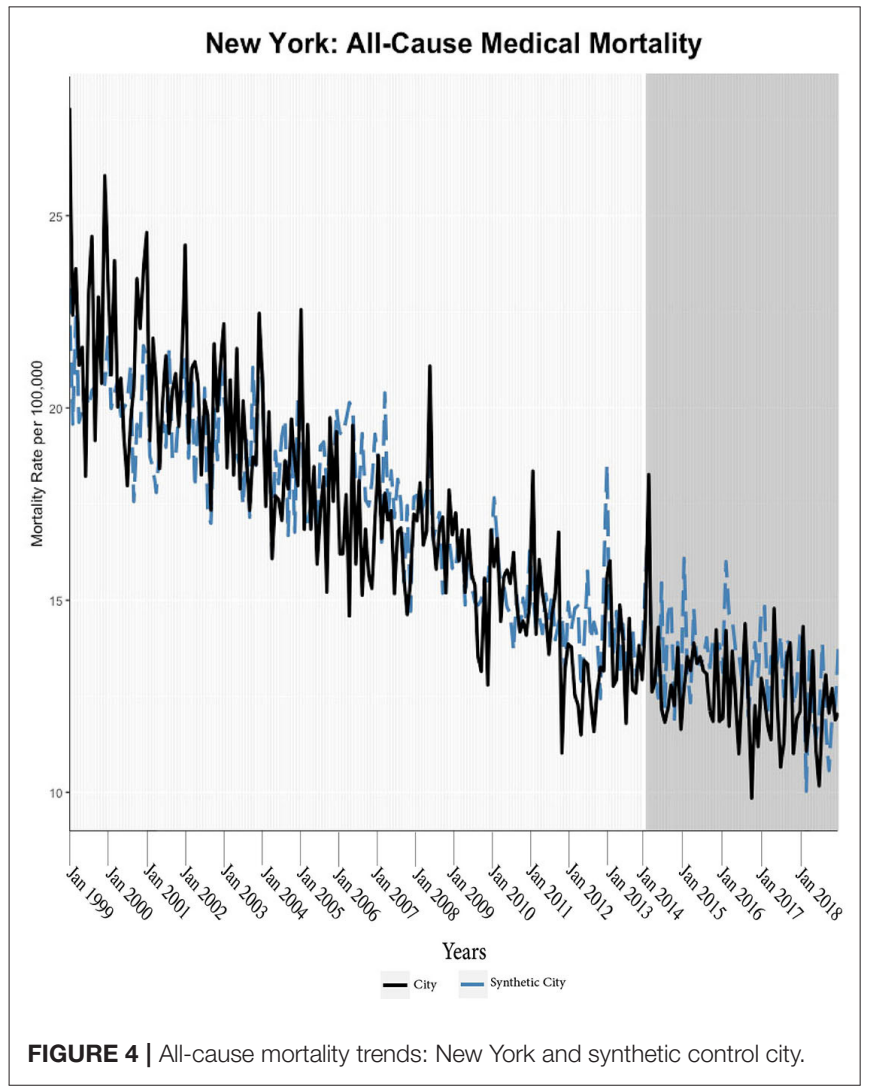

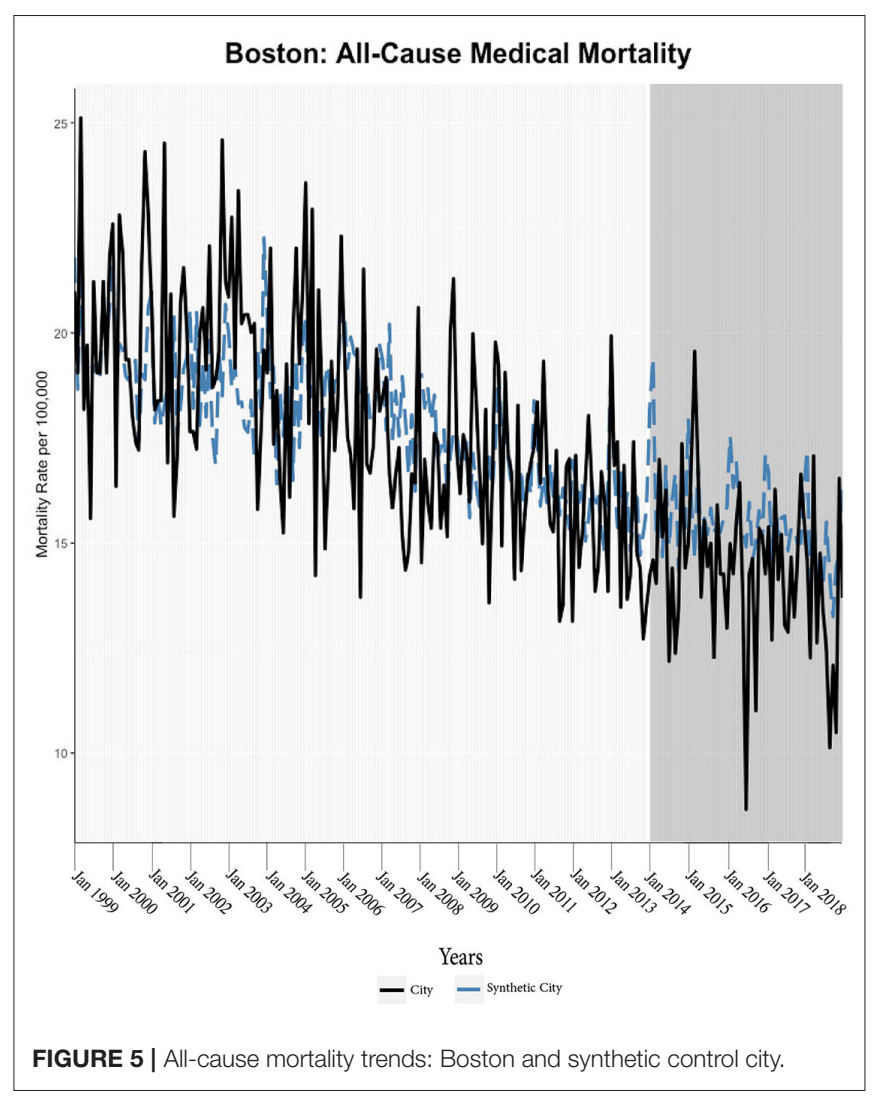

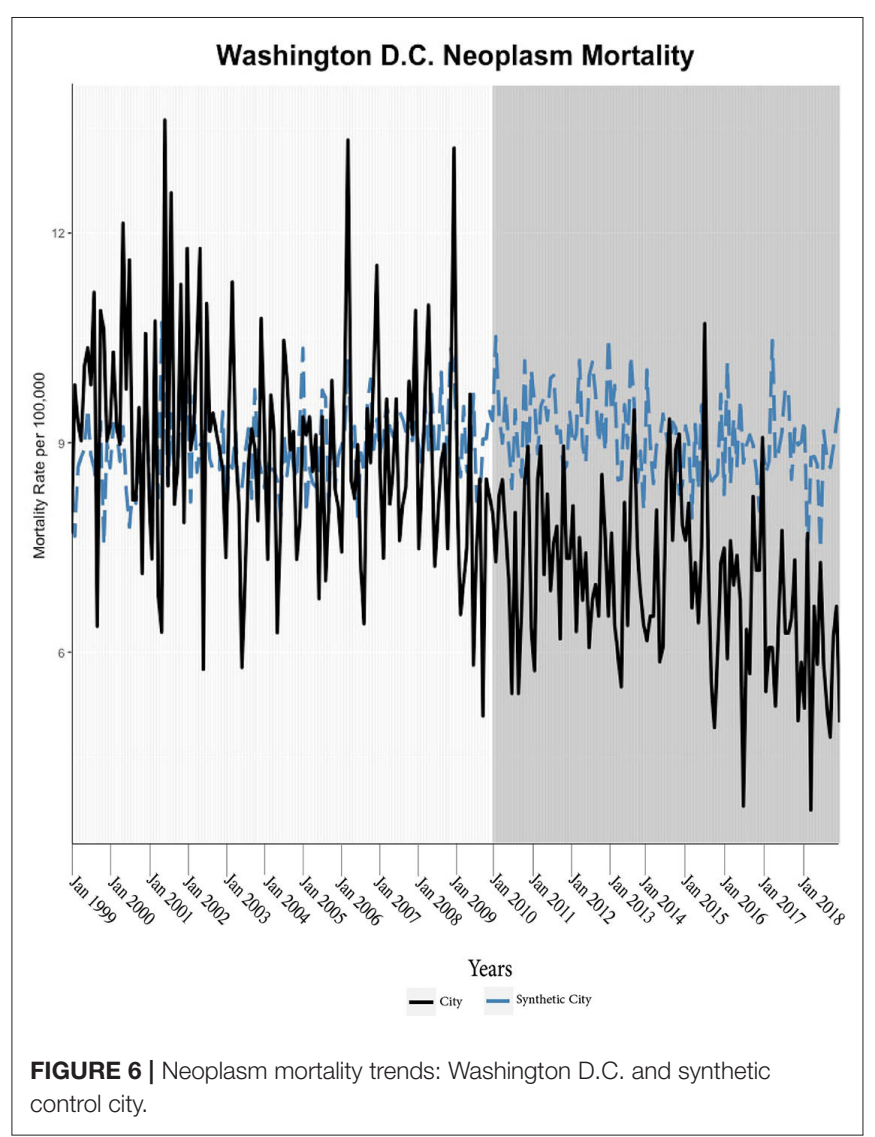




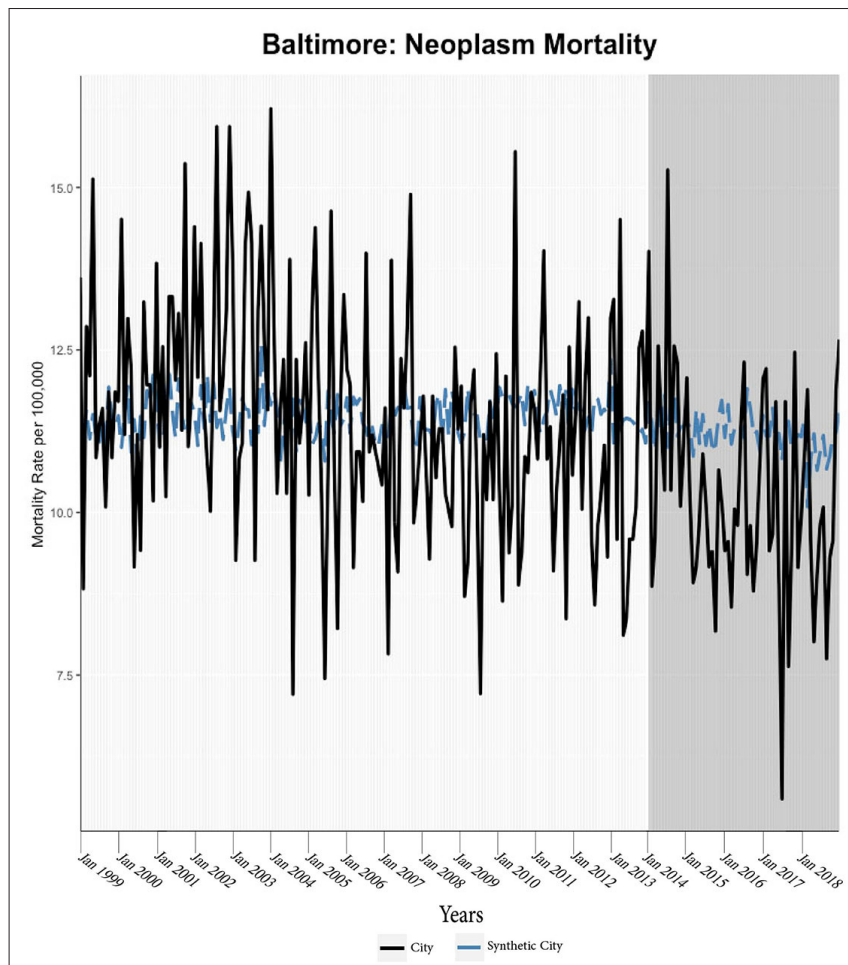

FIGURE 7 | Neoplasm mortality trends: Baltimore and synthetic control city.

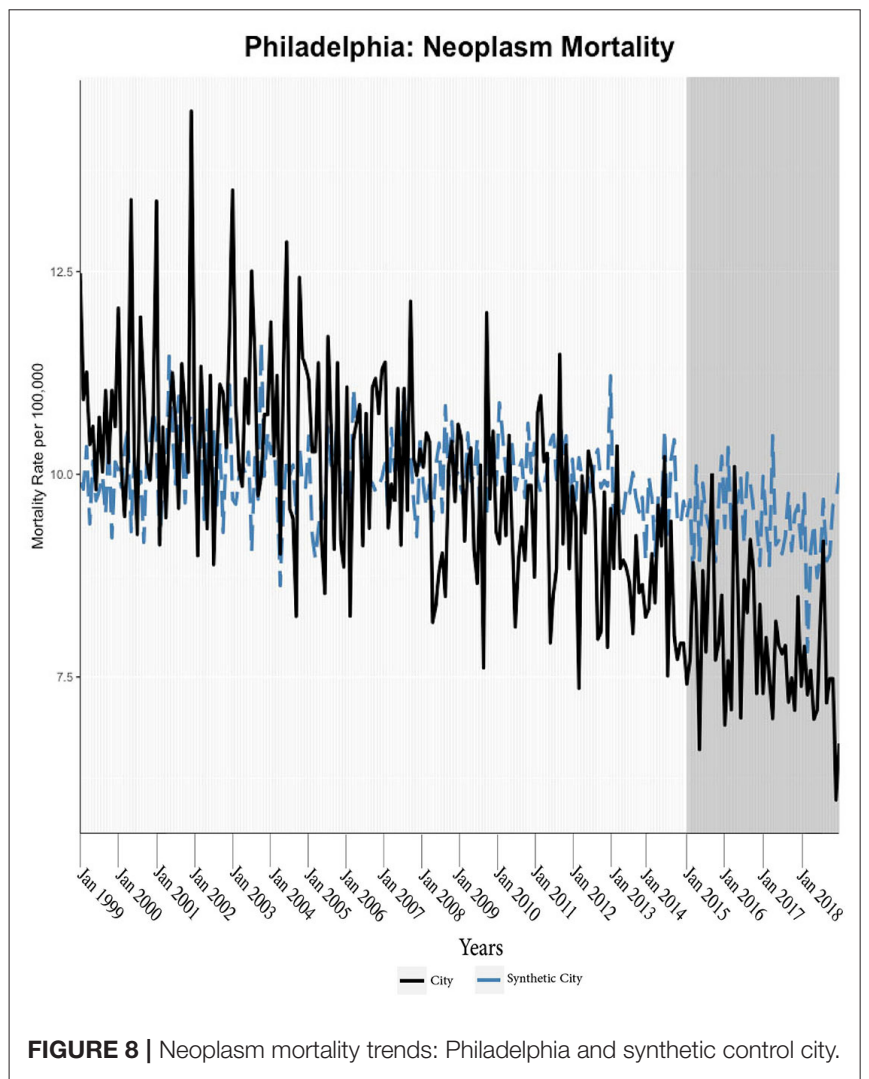

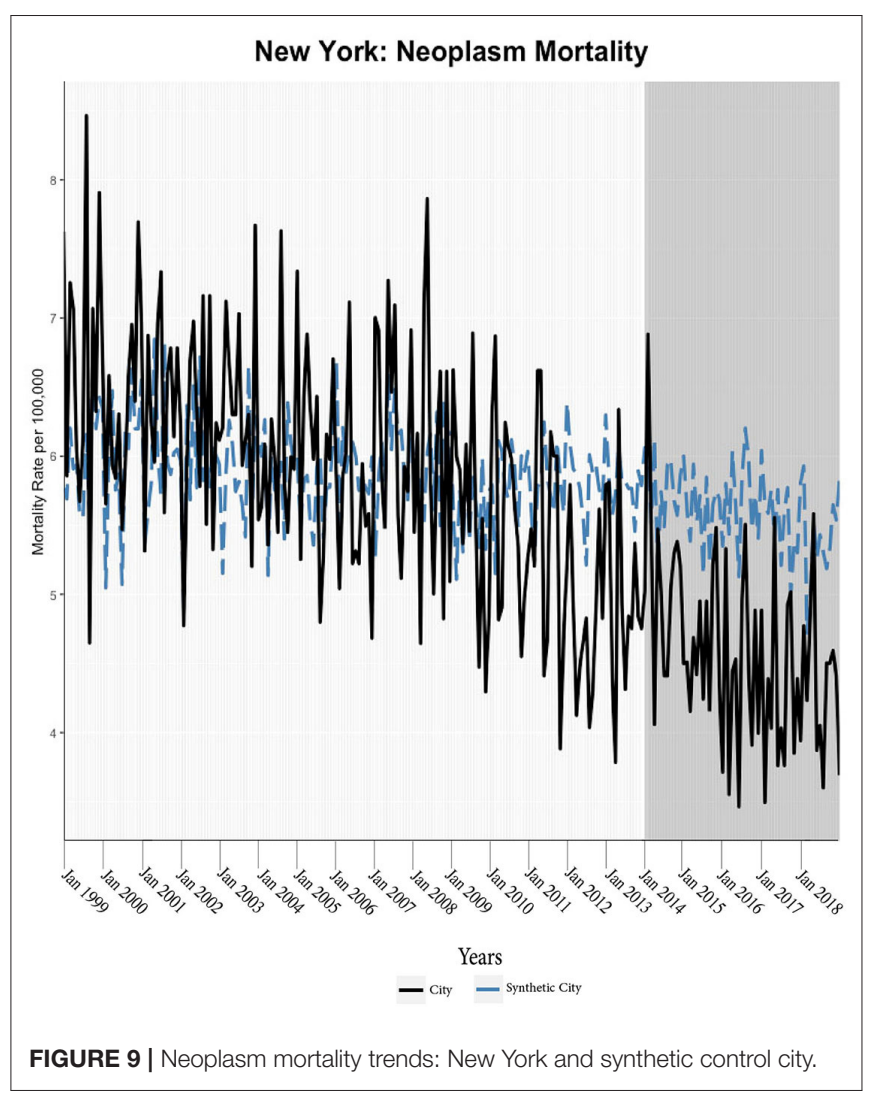

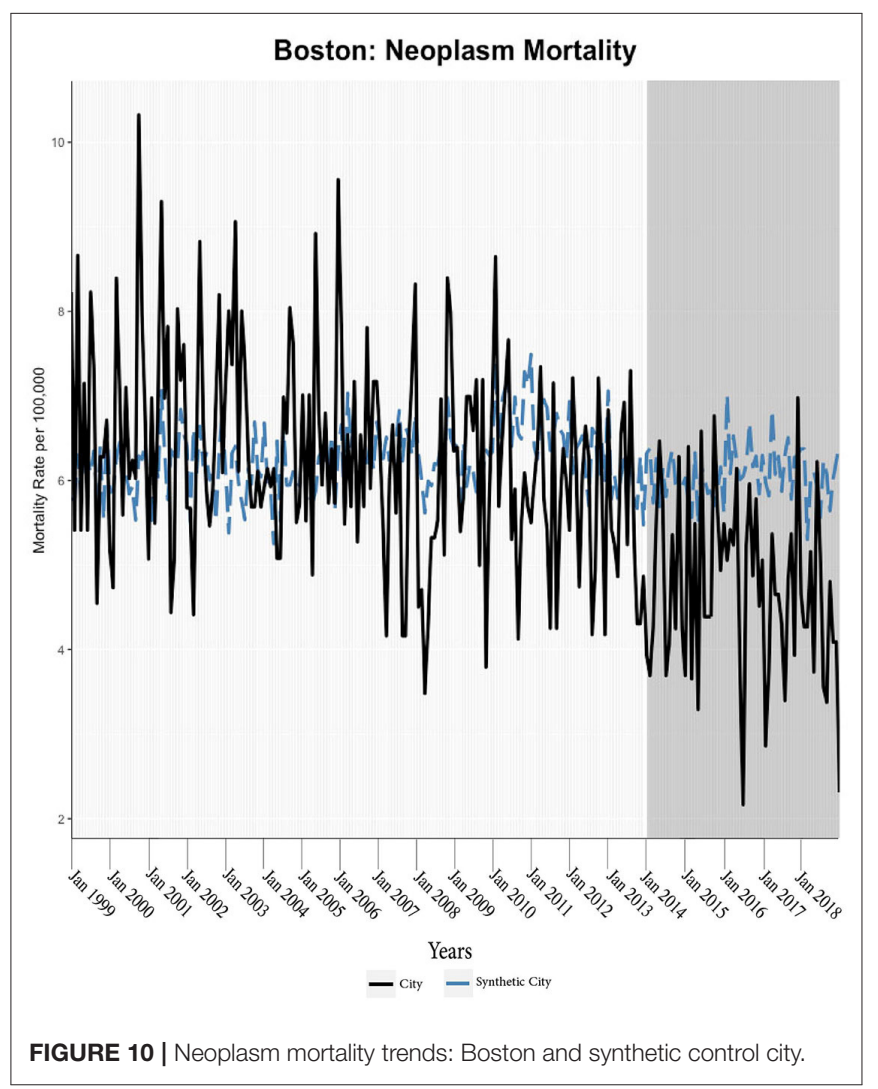


effect sizes are seen across all cities (ATT range $=-1.39$ to -2.78 ) apart from Washington D.C., which saw a much larger decrease $($ ATT $=-5.40)$. The -5.40 ATT value for Washington D.C. conveys that the average monthly neoplasm mortality rate per 100,000 was reduced by 5.40 over the post-intervention period. When assessing neoplasms, we observe a significant reduction in mortality in four cities excluding New York (Figures 510). Pooled across all cities, there was a significant reduction in the neoplasm (Population-Adjusted ATT $=-1.37[95 \%$ CI $-2.73,-0.42]$ ) and all-cause medical (Population-Adjusted $\mathrm{ATT}=-2.57$ [95\% CI $-8.46,-0.58])$ mortality rates but not cardiovascular (Population-Adjusted ATT $=-3.79$ [95\% CI $-24.57,11.68]$ ) and respiratory mortality (Population-Adjusted $\mathrm{ATT}=-0.91[95 \% \mathrm{CI}-6.99,4.89]$ ) (see Figures $12-22$ in the Appendix).

According to established practices, we discard models that have grossly mismatched pre-intervention mortality trends between the treatment and synthetic units (27); this was only observed for respiratory mortality in Baltimore, which was highly volatile. Subsequently, the treatment effect was calculated through the ATT and CTT parameters; $p$-values indicated levels of statistical significance (Table 2; Figure 11). Parameters (ATT, \%ATT, or CTT), time unit of measurement (yearly or monthly), and post-intervention period length must be considered when comparing across the reported values. Overall, our synthetic control models indicate a trend of decreases in neoplasm and all-cause medical mortality rates in cities that expanded Medicaid compared to their estimated counterfactuals. Sensitivity analyses of monthly data converted to yearly data indicated that the analysis was not sensitive to yearly data. Specifically, the conversion of monthly all-cause medical and neoplasm mortality into yearly aggregates changed significant differences into insignificant differences (see Tables 1, 2 in the Appendix). The intime placebo sensitivity analysis indicated that the false intervention time point did not result in significant change in mortality rates (see Table 3 and Figures $11-20$ in the Appendix). The weighted composition of each synthetic control model in the mortality analyses is included (see Table 4 in Appendix).

The generalized synthetic control analyses of insurance rates indicated that the observed city after Medicaid expansion were all much greater than predicted (Table 3) (see Figures 21-25 in Appendix). Most notably, the gains in Washington D.C. (ATT $=4.23 \%, p=0.050)$, Baltimore $(\mathrm{ATT}=3.23 \%, p=0.048)$, and Philadelphia (ATT $=4.30 \% p=0.046$ ) were significantly greater than the predicted trends in the absence of Medicaid expansion. New York and Boston, two cities with already had high rates of coverage prior to Medicaid expansion and the derived treatment effects did not significantly differ from the predicted gains.

\section{DISCUSSION AND CONCLUSION}

Three principal conclusions can be drawn from our analysis. First, we document significant declines in all-cause medical mortality, partly driven by concurrent and significant reductions in neoplasm mortality, in most Northeastern cities following
ACA Medicaid expansion. Second, while Medicaid expansion does appear to be associated with reductions in mortality rates across the cities, we observe evidence of varied effects. Third, analyses of yearly data in this study generally lacked sufficient statistical power.

Most cities experienced significant decreases in neoplasm mortality and all-cause medical mortality after Medicaid expansion as compared to the predicted rates. The potential mechanisms of this observed decline may be several. An intended function of Medicaid expansion was to decrease the uninsured rate $(15,16)$. While there was uptake of Medicaid coverage, much of this enrollment was moderate followed by a mild and steady increase (11). While the magnitude of increased insurance coverage may appear minor, more ill and vulnerable populations (e.g., women, African Americans, Hispanics, immigrants) gained coverage through Medicaid expansion which may explain the larger decrease in mortality observed in this study $(11,28)$.

New enrollees encountered reduced financial barriers which led to receiving timelier clinical and surgical care along with better ability to access prescription medicines for acute and chronic conditions (29-33). Improved self-reported and objective measures of quality as a result of Medicaid expansion accompanied increasing use of preventive care (34). In totality, Medicaid expansion reduced financial barriers and improved integration of communities in medical systems which resulted in individuals receiving more necessary and high-quality medical care (34). Additionally, Medicaid expansion has been associated with increased overall financial stability $(35,36)$. Greater financial stability, especially for those managing chronic conditions or suffering catastrophic events, not only allows for appropriate seeking of care but also may lead to broader health benefits. For example, families with greater financial stability are more able to achieve higher standards of living which act through material and psychosocial mechanisms to produce superior wellbeing $(12,37)$.

Considering neoplasm mortality, long standing research has generally considered the inability to access quality medical care as the major determinant of mortality especially in populations with lower socioeconomic status (37). Numerable studies of those with newfound access to Medicaid indicated that these individuals benefited greatly from expansion in the context of cancer care and mortality (38-42). As disease stage influences neoplasm prognosis, access to care is naturally a significant influence on neoplasm mortality (37). The longitudinal nature of oncological disease would suggest that reductions in mortality should become more profound as time passes from Medicaid expansion. Nevertheless, more proximal effects on cancer mortality due to the newfound ability to access diagnostic care and treatment have been reproduced not only in this study but also in others. Those with undiagnosed cancers were found to receive earlier screenings and diagnostic tests leading to appropriate oncological intervention $(38,42)$. Examining data as early as three-years after Medicaid expansion, Lin et al. found a reduction of late-stage lung cancer diagnoses and an increase in early-stage lung cancer diagnoses (41). Lung cancer as well as other aggressive forms of cancer may progress rapidly 
TABLE 2 | Treatment effects on mortality rates in treated cities.

\begin{tabular}{|c|c|c|c|c|c|c|c|}
\hline & ATT & Lower 95\% Cl & Higher $95 \% \mathrm{Cl}$ & ATT $^{\dagger}{ }^{+}$ & ATT\% Lower 95\% Cl & АTT\% Higher $95 \%$ CI & $p$ \\
\hline \multicolumn{8}{|l|}{ Washington D.C. | 2010} \\
\hline Circulatory mortality & -14.83 & -45.20 & 7.47 & -13.62 & -41.51 & 6.86 & 0.168 \\
\hline Respiratory mortality & 2.31 & -6.21 & 9.73 & 18.39 & -49.41 & 77.43 & 0.658 \\
\hline Neoplasm mortality & -1.95 & -3.04 & -0.98 & -21.88 & -34.10 & -10.99 & $0.002^{\star \star \star}$ \\
\hline All-cause medical mortality & -5.40 & -12.50 & -3.34 & -18.84 & -43.64 & -11.67 & $<0.001^{\star \star *}$ \\
\hline \multicolumn{8}{|l|}{ Baltimore | 2014} \\
\hline Circulatory mortality & -7.00 & -24.83 & 3.13 & -4.35 & -15.43 & 1.95 & 0.148 \\
\hline Respiratory mortality & 1.96 & -2.99 & 6.39 & 6.78 & -10.33 & 22.08 & 0.482 \\
\hline Neoplasm mortality & -0.96 & -1.90 & -0.17 & -8.56 & -17.05 & -1.53 & $0.01^{\star \star \star}$ \\
\hline All-cause medical mortality & -1.76 & -6.51 & -0.28 & -4.48 & -16.53 & -0.70 & $0.038^{\star \star}$ \\
\hline \multicolumn{8}{|l|}{ Philadelphia | 2015} \\
\hline Circulatory mortality & 0.96 & -13.22 & 9.47 & 0.86 & -11.89 & 8.51 & 0.934 \\
\hline Respiratory mortality & -1.37 & -5.10 & 1.43 & -5.95 & -22.21 & 6.22 & 0.336 \\
\hline Neoplasm mortality & -1.63 & -2.73 & -0.99 & -17.17 & -28.72 & -10.39 & $<0.001^{\star \star \star}$ \\
\hline All-cause medical mortality & -1.97 & -5.92 & -0.50 & -6.77 & -20.29 & -1.71 & $0.018^{\star \star}$ \\
\hline \multicolumn{8}{|l|}{ New York | 2014} \\
\hline Circulatory mortality & -0.39 & -25.30 & 19.57 & -0.87 & -56.70 & 43.86 & 0.802 \\
\hline Respiratory mortality & -3.00 & -10.44 & 4.42 & -26.77 & -93.16 & 39.48 & 0.322 \\
\hline Neoplasm mortality & -1.03 & -2.86 & 0.203 & -34.46 & -95.69 & 6.77 & $0.096^{\star}$ \\
\hline All-cause medical mortality & -2.78 & -10.65 & -0.13 & -18.11 & -69.29 & -0.86 & $0.04^{\star \star}$ \\
\hline \multicolumn{8}{|l|}{ Boston | 2014} \\
\hline Circulatory mortality & -7.33 & -18.72 & 2.50 & -15 & -38.31 & 5.12 & 0.144 \\
\hline Respiratory mortality & -0.67 & -5.19 & 4.68 & -6.07 & -47.09 & 42.46 & 0.836 \\
\hline Neoplasm mortality & -1.35 & -2.49 & -0.55 & -22.17 & -40.93 & 9.06 & $0.004^{\star \star \star}$ \\
\hline All-cause medical mortality & -1.39 & -4.53 & 0.09 & -8.85 & -28.77 & 0.57 & $0.068^{\star}$ \\
\hline
\end{tabular}

${ }^{*} \leq 0.10^{* \star} \leq 0.05^{* * \star} \leq 0.01$.

${ }^{\dagger}$ Percentage change of post-intervention mortality of Medicaid expansion city from synthetic control prediction.

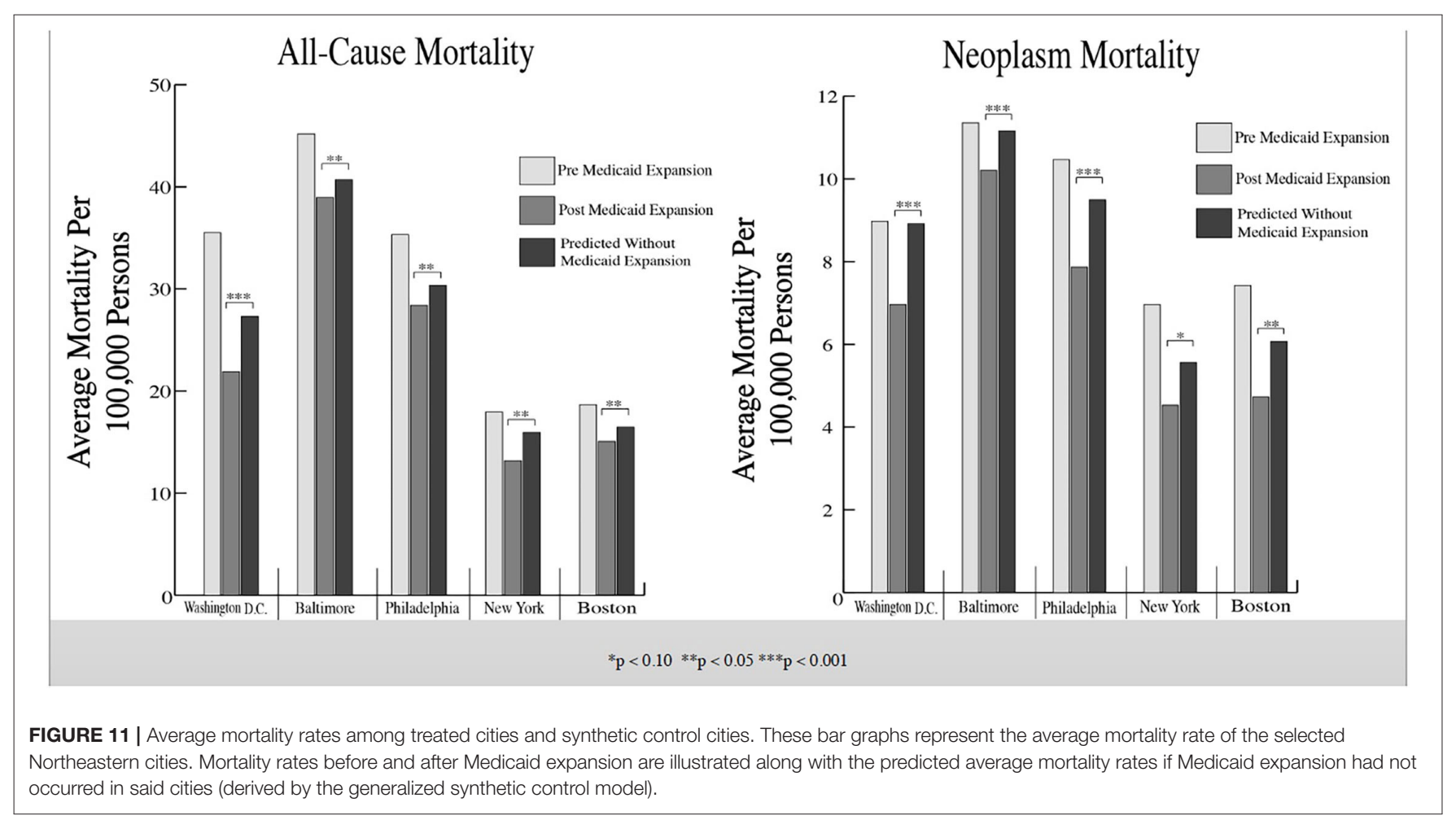


TABLE 3 | Treatment effects on percentage of population insured in treated cities.

\begin{tabular}{lcccc}
\hline City | Intervention year & ATT & Lower 95\% Cl & Higher 95\% Cl & $\boldsymbol{p}$ \\
\hline Washington D.C. | 2010 & $4.22 \%$ & $0.28 \%$ & $10.58 \%$ & $0.048^{\star *}$ \\
Baltimore | 2014 & $3.23 \%$ & $0.30 \%$ & $10.56 \%$ & $0.046^{\star *}$ \\
Philadelphia | 2015 & $4.31 \%$ & $0.24 \%$ & $6.66 \%$ & $0.036^{\star *}$ \\
New York | 2014 & $0.86 \%$ & $-13.13 \%$ & $9.75 \%$ & 0.860 \\
Boston | 2014 & $-12.54 \%$ & $-16.91 \%$ & $7.80 \%$ & 0.618 \\
\hline${ }^{* \star} \leq 0.05$ & & & &
\end{tabular}

leading to appreciable mortality that can be avoided by early diagnosis as potentially related to Medicaid expansion (41).

Neoplasm mortality reductions partly, but not fully, underlie all-cause medical mortality reductions; unrelated reductions may result from other causes that are amenable to increased access; these may or may not have been captured separately in our study.

Variations in the observed effect sizes between cities may be related to distinct policy landscapes before Medicaid expansion. Boston, for example, passed the MHCR in 2006 and this already offered coverage to many near FLP. Indeed, baseline mortality rates in Boston were lower than in other cities in our study prior to Medicaid expansion. Further, extensive preMedicaid expansion insurance coverage and limited baseline mortality suggest that ACA Medicaid expansion impacts be minimal in Boston. Similarly, New York City had implemented state-wide low-income coverage policies for non-elderly adults prior to the formal expansion of Medicaid in 2014 (43). Specifically, the 2001 Family Health Plus program was expanded to $100 \%$ of FLP (43); this standing program may have reduced the impact of Medicaid expansion in New York City. Such characterization of these two policy landscapes is bolstered by the results of the insurance rate analysis. Specifically, New York and Boston did not observe significant gains in the insured population as compared to their predicted trajectory absent Medicaid expansion. Nevertheless, we do observe smaller and statistically insignificant gains in the insured rate after Medicaid Expansion; given the already high rates, we anticipate that only minor gains in insurance rates would have been possible, so that this limited magnitude may not achieve statistical significance.

By contrast, the remaining cities resided in states where Medicaid expansion resulted in a large expansion in their public insurance eligibility criteria. Before its early expansion of Medicaid in 2010, Washington D.C. had only adopted narrow programs beyond the federal Medicaid requirements. Washington D.C. not only expanded Medicaid early, but the district set eligibility criteria to $210 \%$ FPL, which makes this expansion the largest of any included city (44). Similarly, Pennsylvania had heavily utilized waivers to create several targeted programs but none of these programs were broadly applicable to Medicaid expansion-eligible populations. Philadelphia had similar mortality rates to Washington D.C. but the extent of Medicaid expansion was less significant (i.e., $138 \%$ FPL).
Unlike Washington D.C. and Philadelphia, the 2006 Primary Adult Care (PAC) program under the HealthChoice program in Baltimore expanded coverage (prescription, primary care, behavioral health) to childless adults at or below $116 \%$ FPL (45). Accordingly, Medicaid expansion did moderately expand Baltimore's coverage criteria from baseline. Nevertheless, Washington D.C. and Baltimore had the highest mortality rates before Medicaid expansion; this inclines both cities to larger observed effects. Medicaid expansion operated in the context of Baltimore's elevated mortality rates, higher poverty rates, and large minority populations; these factors likely promote greater Medicaid expansion effects. The aforementioned factors suggest that Medicaid expansion might be most efficacious in Washington D.C., Baltimore and Philadelphia. The insurance rate models found significant increases in the insured percentage of said cities as compared to those trends predicted in the absence of Medicaid expansion. This provides evidence that Medicaid expansion may have increased insurance rates significantly.

Importantly, our findings diverge from state-wide analyses, in that, the magnitude of Medicaid expansion impact was larger in urban areas than in states as a whole. We attribute this to multiple factors. First, the concentration of health issues in urban areas may be more able to capture the health effects of Medicaid expansion (21). Second, greater proximity to medical care in urban environments, unlike rural areas, suggests that financial access is a stronger variable in determining healthcare access (46). With respect to Medicaid expansion specifically, previous studies have shown that medical utilization starkly increased in states after the introduction of Medicaid expansion (28). Such observed increases in utilization along with broader increases in physician supply and particularly high densities of healthcare resources in large cities strongly suggest that the increased access to care was achieved through Medicaid coverage $(28,46)$. This further suggests that lesser gains in urban coverage, as compared to rural populations, can be potent in improving population health in urban settings (47).

Certain limitations should be considered. Sensitivity analyses indicated that yearly data were unable to derive similarly statistically significant treatment effects as compared to monthly data. This may be explained by differences in granularity reducing study power, seasonal trends, etc. Thus, it is likely that the yearly circulatory and respiratory mortality data used are inadequate to capture treatment effects. Otherwise, mortality is an extreme marker of population health not fully representative of the total effects of Medicaid expansion. Considering trend volatility, dissimilar baseline mortality, different intervention sizes, and the fundamental limitations in the GSC, lack of findings should not be considered as precluding effects on certain types of mortality (e.g., respiratory).

Like most other Medicaid expansion studies, this study fundamentally compares mortality rates between non-ME and Medicaid expansion states where these groups may experience unique confounding influences given the ecological nature of the data. For instance, city specific events related 
to police brutality, weather events, civil unrest, etc., may be lay the ground for specific forms of medical-related mortality via more diffuse factors (e.g., stress, slow medical emergency response times). Nevertheless, these effects may not strongly affect the sum magnitude of the observed mortality and would likely only dampen observed degree effects attributed to Medicaid expansion in the present study. The use of only urban populations, numerous independent relevant covariates, and the generalized synthetic control method allow this study to limit the influence of observed and unobserved confounding unlike most previous studies of Medicaid expansion. Regardless, overfitting is a concern in synthetic control models and while the GSC model does improve upon overfitting issues found in the traditional synthetic control methods via the use of semiparametric estimation and cross-validation schema, the risk of overfitting remains (25).

Unlike most literature, the county-level nature of this analysis limits the number of individuals available for study. As such, separate analyses of older sub-populations were inviable given the limited size of such a sub-population and the risk of stochastic variability biasing the model. Nevertheless, the inclusion of younger and healthier populations in the presented results more likely underestimates the treatment effects of Medicaid expansion (28). Similarly, the nature of the data utilized county-wide covariates, as such analyses of mortality rates in subsets of particular covariate categories (e.g., education-level) was unable to be conducted. Further studies are required to determine whether the observed effects of Medicaid expansion can be generalized to other cities without Medicaid expansion. To this point, each city's results must be considered

\section{REFERENCES}

1. Blumenthal D, Abrams M, Nuzum R. The affordable care act at 5 years. $N$ Engl J Med. (2015) 372:2451-8. doi: 10.1056/NEJMhpr1503614s

2. Jacobs LR, Callaghan T. Why states expand medicaid: Party, resources, and history. J Health Polit Policy Law. (2013) 38:1023-50. doi: 10.1215/03616878-2334889

3. Kaestner R, Garrett B, Chen J, Gangopadhyaya A, Fleming C. Effects of ACA medicaid expansions on health insurance coverage and labor supply. J Policy Anal Manage. (2017) 36:608-42. doi: 10.1002/pam.21993

4. Isola S, Reddivari AKR. Affordable Care Act. [Updated 2021 Jul 15]. In: StatPearls [Internet]. Treasure Island, FL: StatPearls Publishing (2021).

5. Goodman-Bacon A. Public insurance and mortality: evidence from medicaid implementation. J Polit Econ. (2018) 126:216-62. doi: 10.1086/695528

6. Currie J, Gruber J. Health insurance eligibility, utilization of medical care, and child health. Q J Econ. (1996) 111:431-66. doi: 10.2307/2946684

7. Howell EM. The impact of the Medicaid expansions for pregnant women: a synthesis of the evidence. Med Care Res Rev. (2001) 58:330. doi: 10.1177/107755870105800101

8. Sommers BD, Long SK, Baicker K. Changes in mortality after Massachusetts health care reform: a quasi-experimental study. Ann Intern Med. (2014) 160:585-93. doi: 10.7326/M13-2275

9. Powell D. Imperfect Synthetic Controls: Did the Massachusetts Health Care Reform Save Lives? (2018).

10. Sommers BD. State medicaid expansions and mortality, revisited: a cost-benefit analysis. Am J Health Econ. (2017) 3:392-421. doi: 10.1162/ajhe_a_00080 given the unique treatment intensity and policy history characterized above.

Significant reductions in multiple forms of urban mortality were attributed to Medicaid expansion. The degree of effects was seemingly related to baseline mortality rates, prior expansion status, and the magnitude of Medicaid expansion. Our study indicates that Medicaid expansion saved lives in the included urban settings.

\section{DATA AVAILABILITY STATEMENT}

The original contributions presented in the study are included in the article/Supplementary Material, further inquiries can be directed to the corresponding author.

\section{AUTHOR CONTRIBUTIONS}

CA designed and conducted the initial gathering of data, organization, analysis, and organized the findings and subsequently put the information in a manuscript format. PP was involved in the data analysis, figure making, and the writing of the manuscript. SA was involved in the organization and the writing of the manuscript. All authors contributed to the article and approved the submitted version.

\section{SUPPLEMENTARY MATERIAL}

The Supplementary Material for this article can be found online at: https://www.frontiersin.org/articles/10.3389/fpubh. 2021.707907/full\#supplementary-material

11. Finkelstein A, Taubman S, Wright B, Bernstein M, Gruber J, Newhouse JP, et al. The Oregon health insurance experiment: evidence from the first year. $Q$ J Econ. (2012) 127:1057-106. doi: 10.1093/qje/qjs020

12. Borgschulte M, Vogler J. Did the ACA medicaid expansion save lives? J Health Econ. (2020) 72:102333. doi: 10.1016/j.jhealeco.2020.102333

13. Black B, Hollingsworth A, Nunes L, Simon K. The Effect of Health Insurance on Mortality: Power Analysis and What We Can Learn from the Affordable Care Act Coverage Expansions. National Bureau of Economic Research (2019). p. 0898-2937.

14. Miller S, Altekruse S, Johnson N, Wherry LR. Medicaid and Mortality: New Evidence from Linked Survey and Administrative Data. National Bureau of Economic Research (2019). p. 0898-2937.

15. Bhatt CB, Beck-Sagué CM. Medicaid expansion and infant mortality in the United States. Am J Pubicl Health. (2018) 108:565-67. doi: 10.2105/AJPH.2017.304218

16. Eliason EL. Adoption of Medicaid Is Associated with Lower Maternal Mortality. Women's Health Issues (2020). 30:147-52. doi: 10.1016/j.whi.2020. 01.005

17. Khatana SAM, Bhatla A, Nathan AS, Giri J, Shen C, Kazi DS, et al. Association of Medicaid expansion with cardiovascular mortality. JAMA Cardiol. (2019) 4:671-79. doi: 10.1001/jamacardio.2019.1651

18. Swaminathan S, Sommers BD, Thorsness R, Mehrotra R, Lee Y, Trivedi AN. Association of Medicaid expansion with 1-year mortality among patients with end-stage renal disease. JAMA. (2018) 320:2242-50. doi: 10.1001/jama.2018.16504

19. Freudenberg N. Time for a national agenda to improve the health of urban populations. Am J Publ Health. (2000) 90:837. doi: 10.2105/AJPH.90.6.837 
20. Geronimus AT. To mitigate, resist, or undo: addressing structural influences on the health of urban populations. Am J Public Health. (2000) 90:867. doi: 10.2105/AJPH.90.6.867

21. Vlahov D, Freudenberg N, Proietti F, Ompad D, Quinn A, Nandi V, et al. Urban as a determinant of health. J Urban Health. (2007) 84:1626. doi: 10.1007/s11524-007-9169-3

22. Ompad DC, Galea S, Caiaffa WT, Vlahov D. Social determinants of the health of urban populations: methodologic considerations. J Urban Health. (2007) 84:42-53. doi: 10.1007/s11524-007-9168-4

23. Vafaei A, Rosenberg MW, Pickett W. Relationships between income inequality and health: a study on rural and urban regions of Canada. Rural Remote Health. (2010) 10:1430.

24. Day JC. Rates of uninsured fall in rural counties, remain higher than Urban counties. Census.gov. (2021). Available online at: https://www.census. gov/library/stories/2019/04/health-insurance-rural-america.html (accessed November 3, 2021).

25. Xu Y. Generalized synthetic control method: causal inference with interactive fixed effects models. Polit Anal. (2017) 25:57-76. doi: 10.1017/pan.2016.2

26. O’Neill S, Kreif N, Sutton M, Grieve R. A comparison of methods for health policy evaluation with controlled pre-post designs. Health Serv Res. (2020) 55:328-38. doi: 10.1111/1475-6773.13274

27. Abadie A, Diamond A, Hainmueller J. Comparative politics and the synthetic control method. Am J Polit Sci. (2015) 59:495-510. doi: 10.1111/ajps.12116

28. Sommers BD, Blendon RJ, Orav EJ, Epstein AM. Changes in utilization and health among low-income adults after Medicaid expansion or expanded private insurance. JAMA Intern Med. (2016) 176:1501-9. doi: 10.1001/jamainternmed.2016.4419

29. Chou SC, Gondi S, Weiner SG, Schuur JD, Sommers BD. Medicaid expansion reduced emergency department visits by low-income adults due to barriers to outpatient care. Med Care. (2020) 58:511-8. doi: 10.1097/MLR.0000000000001305

30. Adamson BJ, Cohen AB, Estevez M, Magee K, Williams E, Gross CP, et al. Affordable Care Act (ACA) medicaid expansion impact on racial disparities in time to cancer treatment. Am Soc Clin Oncol. (2019) 27. doi: 10.1200/JCO.2019.37.18_suppl.LBA1

31. Mahendraratnam N, Dusetzina SB, Farley JF. Prescription drug utilization and reimbursement increased following state Medicaid expansion in 2014. J Manag Care Spec Pharm. (2017) 23:355-63. doi: 10.18553/jmcp.2017.23 .3 .355

32. Loehrer AP, Chang DC, Scott JW, Hutter MM, Patel VI, Lee JE, et al. Association of the affordable care act medicaid expansion with access to and quality of care for surgical conditions. JAMA Surg. (2018) 153:e175568. doi: 10.1001/jamasurg.2017.5568

33. Lin S, Brasel KJ, Chakraborty O, Glied SA. Association between medicaid expansion and the use of outpatient general surgical care among US adults in multiple states. JAMA Surg. (2020) 155:1058-66. doi: 10.1001/jamasurg.2020.2959

34. Cole MB, Galárraga O, Wilson IB, Wright B, Trivedi AN. At federally funded health centers, medicaid expansion was associated with improved quality of care. Health Aff. (2017) 36:40-8. doi: 10.1377/hlthaff.2016.0804

35. Allen HL, Eliason E, Zewde N, Gross T. Can medicaid expansion prevent housing evictions? Health Aff. (2019) 38:14517. doi: 10.1377/hlthaff.2018.05071

36. Hu L, Kaestner R, Mazumder B, Miller S, Wong A. The effect of the affordable care act Medicaid expansions on financial wellbeing. J Public Econ. (2018) 163:99-112. doi: 10.1016/j.jpubeco.2018.04.009
37. Wherry LR, Miller S, Kaestner R, Meyer BD. Childhood medicaid coverage and later-life health care utilization. Rev Econ Stat. (2018) 100:287302. doi: 10.1162/REST_a_00677

38. Takvorian SU, Oganisian A, Mamtani R, Nandi M, Shulman LN, Bekelman JE, et al. Association of medicaid expansion under the affordable care act with insurance status, cancer stage, and timely treatment among patients with breast, colon, and lung cancer. JAMA Netw Open. (2020) 3:e1921653. doi: 10.1001/jamanetworkopen.2019.21653

39. $\mathrm{Fu} \mathrm{S}$, Rose L, Knowlton L. The affordable care act and insurance status, stage, and timely treatment among patients with cancer: what are the possible effects? JAMA Netw Open. (2020) 3:e192169. doi: 10.1001/jamanetworkopen.2019.21690

40. Dawes AJ, Louie R, Nguyen DK, Maggard-Gibbons M, Parikh P, Ettner $\mathrm{SL}$, et al. The impact of continuous medicaid enrollment on diagnosis, treatment, and survival in six surgical cancers. Health Serv Res. (2014) 49:1787-811. doi: 10.1111/1475-6773.12237

41. Lin L, Soni A, Sabik LM, Drake C. Early- and late-stage cancer diagnosis under 3 years of medicaid expansion. Am J Prev Med. (2021) 60:1049. doi: 10.1016/j.amepre.2020.06.020

42. Hendryx M, Luo J. Increased cancer screening for low-income adults under the affordable care act medicaid expansion. Med Care. (2018) 56:944949. doi: 10.1097/MLR.0000000000000984

43. Black LI, Schiller JS. State variation in health care service utilization: United States, 2014. NCHS Data Brief. (2016) 245:1-8.

44. Sommers BD, Arntson E, Kenney GM, Epstein AM. Lessons from early medicaid expansions under health reform: interviews with medicaid officials. Medicare Medicaid Res Rev. (2013) 3:mmrr.003.04.a02. doi: 10.5600/mmrr.003.04.a02

45. Pollak AN, Steffen CB. Study of Mortality Rates of African American Infants and Infants in Rural Areas (n.d.). Available online at: https://mhcc.maryland. gov/mhcc/pages/home/workgroups/documents/african_american_study/ DRAFTRPT4WRKGRP82719.pdf (accessed November, 2021).

46. Hartley D. Rural health disparities, population health, and rural culture. Am J Public Health. (2004) 94:1675-8. doi: 10.2105/AJPH.94.10.1675

47. Soni A, Hendryx M, Simon K. Medicaid expansion under the affordable care act and insurance coverage in rural and urban areas. J Rural Health. (2017) 33:217-26. doi: 10.1111/jrh.12234

Conflict of Interest: The authors declare that the research was conducted in the absence of any commercial or financial relationships that could be construed as a potential conflict of interest.

Publisher's Note: All claims expressed in this article are solely those of the authors and do not necessarily represent those of their affiliated organizations, or those of the publisher, the editors and the reviewers. Any product that may be evaluated in this article, or claim that may be made by its manufacturer, is not guaranteed or endorsed by the publisher.

Copyright (C) 2021 Ayubcha, Pouladvand and Ayubcha. This is an open-access article distributed under the terms of the Creative Commons Attribution License (CC BY). The use, distribution or reproduction in other forums is permitted, provided the original author(s) and the copyright owner(s) are credited and that the original publication in this journal is cited, in accordance with accepted academic practice. No use, distribution or reproduction is permitted which does not comply with these terms. 